\title{
INVERSE SCATTERING FOR SINGULAR POTENTIALS IN TWO DIMENSIONS
}

\author{
ZIQI SUN AND GUNTHER UHLMANN
}

\begin{abstract}
We consider the Schrödinger equation for a compactly supported potential having jump type singularities at a subdomain of $\mathbb{R}^{2}$. We prove that knowledge of the scattering amplitude at a fixed energy, determines the location of the singularity as well as the jump across the curve of discontinuity. This result follows from a similar result for the Dirichlet to Neumann map associated to the Schrödinger equation for a compactly supported potential with the same type of singularities.
\end{abstract}

\section{INTRODUCTION AND STATEMENT OF THE RESULTS}

In this paper we consider the Schrödinger equation for a compactly supported potential, $q$, having jump type singularities at a subdomain of $\mathbb{R}^{2}$. We prove that knowledge of the scattering amplitude at a fixed energy, $\lambda_{0}$, determines the location of the singularity as well as the jump across the curve of discontinuity.

This problem is reduced to the study of the Dirichlet to Neumann map for the Schrödinger operator $-\Delta+q-\lambda_{0}^{2}$ in a bounded domain of $\mathbb{R}^{2}$. (For the application considered here it is enough to consider the domain to be a ball containing the support of $q$.) We prove that in dimension two the Dirichlet to Neumann map for the Schrödinger operator $-\Delta+q-\lambda_{0}^{2}$ determines uniquely the location of the singularity of $q$ as well as its jump across the curve of discontinuity.

The scattering amplitude of a potential $q \in L^{\infty}\left(\mathbb{R}^{n}\right)$ with compact support is defined via the outgoing eigenfunctions. Namely, $\forall \lambda \in \mathbb{R} \backslash 0, \omega \in S^{n-1}$, there exists $\psi_{+}(\lambda, x, \omega)$, solution of

$$
\left(-\Delta+q-\lambda^{2}\right) \psi_{+}=0
$$

satisfying

$$
\psi_{+}=e^{i \lambda x \cdot \omega}+\frac{a_{q}(\lambda, \theta, \omega) e^{i \lambda|x|}}{|x|^{(n-1) / 2}}+O\left(|x|^{-((n-1) / 2+1)}\right)
$$

with $\theta=x /|x|$. The scattering amplitude, $a_{q}(\lambda, \theta, \omega)$, measures the effect of the potential $q$ on plane waves of the form $e^{i \lambda x \cdot \omega}$.

Received by the editors April 19, 1991.

1991 Mathematics Subject Classification. Primary 35R30; Secondary 35J25, 78A25.

Research of first author partly supported by NSF DMS 9015621.

Research of second author partly supported by NSF DMS 8800153 . 
The inverse scattering problem at a fixed frequency $\lambda_{0} \in \mathbb{R} \backslash 0$ is to study the map

$$
q \stackrel{\mathscr{A}_{\lambda_{0}}}{\rightarrow} a_{q, \lambda_{0}}
$$

where

$$
a_{q, \lambda_{0}}(\theta, \omega)=a_{q}\left(\lambda_{0}, \theta, \omega\right) .
$$

In dimensions $n \geq 3$ the map $\mathscr{A}_{\lambda_{0}}$ is formally overdetermined in the sense that we want to determine a function of $n$-variables from a function depending on $2(n-1)$ variables. Injectivity of $\mathscr{A}_{\lambda_{0}}$ was proved by Novikov [No] in this case. This result can also be proven, as we shall indicate below, as a consequence of the global uniqueness result proven by Sylvester and Uhlmann for the Dirichlet to Neumann map $\Lambda_{q-\lambda_{0}^{2}}$ [S-U, I]. See $\S 2$ for more details.

However, injectivity of $\mathscr{A}_{\lambda_{0}}$ is an open question in the 2-dimensional case. The difficulty is that this is a formally determined problem in this case. Injectivity of $\mathscr{A}_{\lambda_{0}}$ for $q$ close to zero was proven by Novikov [No]. Again this result follows from the corresponding result for the Dirichlet to Neumann map, $\Lambda_{q-\lambda_{0}^{2}}$, proven by Sylvester and Uhlmann [S-U, II]. Sun and Uhlmann [Su-U, I] used the results of [Su-U, II] for the Dirichlet to Neumann to prove that $\mathscr{A}_{\lambda_{0}}$ is locally injective near most potentials (an open and dense set of potentials in the $W^{1, \infty}$ topology) and globally injective for pairs of potentials in an open and dense set in the $W^{1, \infty} \times W^{1, \infty}$ topology.

In this paper we consider potentials $q_{j} \in L^{\infty}\left(\mathbb{R}^{2}\right), j=1,2$, with compact support, having jump type singularities across the boundary of a bounded smooth domain $\Omega_{0}^{j} \subset \mathbb{R}^{2}, j=1,2$. More precisely,

Theorem 1. Let $q_{j} \in C^{1}\left(\overline{\Omega_{0}^{j}}\right), q_{j} \in C^{1}\left(\mathbb{R}^{2} \backslash \Omega_{0}^{j}\right), j=1,2$. Also assume that $\left.\left(q_{j}^{+}-q_{j}^{-}\right)\right|_{\partial \Omega_{j}} \neq 0, j=1,2$. If $\mathscr{A}_{\lambda_{0}}\left(q_{1}\right)=\mathscr{A}_{\lambda_{0}}\left(q_{2}\right)$, then $\Omega_{0}^{1}=\Omega_{0}^{2}$ and $\left.\left(q_{1}^{+}-q_{1}^{-}\right)\right|_{\partial \Omega_{0}^{1}}=\left.\left(q_{2}^{+}-q_{2}^{-}\right)\right|_{\partial \Omega_{0}^{2}}$ where $q_{j}^{+}$(resp. $q_{j}^{-}$) denotes the interior (resp. exterior) limit of $q_{j}$ on $\partial \Omega_{0}^{j}$.

We now define the Dirichlet to Neumann map. Assume that $q \in L^{\infty}(\Omega)$, where $\Omega \subseteq \mathbb{R}^{n}$ is a bounded smooth domain. Assume also that $\lambda_{0}^{2} \in \mathbb{R} \backslash 0$ is not a Dirichlet eigenvalue for $-\Delta+q$. Then for every $f \in H^{1 / 2}(\partial \Omega)$ there is a unique solution $u \in H^{1}(\Omega)$ of the Dirichlet problem

$$
\left(-\Delta+q-\lambda_{0}^{2}\right) u=0,\left.\quad u\right|_{\partial \Omega}=f .
$$

Then we define

$$
\Lambda_{q-\lambda_{0}^{2}}(f)=\left.\frac{\partial u}{\partial \nu}\right|_{\partial \Omega}
$$

with $u$ a solution of $(1.5)$ and $\nu$ the outer unit normal to $\partial \Omega$. The inverse problem is then to study the map

$$
q \stackrel{\Lambda}{\rightarrow} \Lambda_{q-\lambda_{0}^{2}} .
$$

Injectivity of $\Lambda$ was proven in [S-U, I] in the case $n \geq 3$ for $q \in C^{\infty}(\Omega)$. This was extended to $q \in L^{\infty}(\Omega)$ in [N-S-U]. This problem is formally overdetermined in this case. For the formally determined 2-dimensional case injectivity of $\Lambda$ was proved in a $W^{1, \infty}(\Omega)$ neighborhood of $q=0$ [S-U, II]. This 
was extended by Sun to potentials close to constant [Su, I]. Recently, Sun and Uhlmann [Su-U, II] proved that $\Lambda$ is injective near most potentials (an open and dense set in $\left.W^{1, \infty}(\Omega)\right)$. In this paper we prove

Theorem 2. Let $q_{j} \in L^{\infty}(\Omega)$ where $\Omega$ is a bounded smooth domain in $\mathbb{R}^{2}$ and

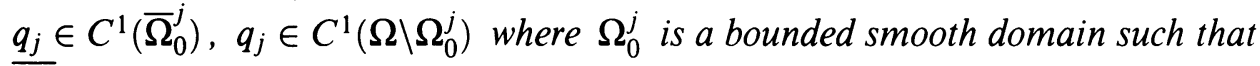
$\overline{\Omega_{0}^{j}} \subset \Omega, j=1,2$. Assume that $\lambda_{0}^{2} \in \mathbb{R}$ is not an eigenvalue for $-\Delta+q_{j}$, and $\left.\left(q_{j}^{+}-q_{j}^{-}\right)\right|_{\partial \Omega_{0}^{j}} \neq 0, j=1,2$. If

$$
\Lambda_{q_{1}-\lambda_{0}^{2}}=\Lambda_{q_{2}-\lambda_{0}^{2}}
$$

then

$$
\Omega_{0}^{1}=\Omega_{0}^{2} \text { and }\left.\left(q_{1}^{+}-q_{1}^{-}\right)\right|_{\partial \Omega_{0}^{1}}=\left.\left(q_{2}^{+}-q_{2}^{-}\right)\right|_{\partial \Omega_{0}^{2}}
$$

with $q_{j}^{ \pm}$defined as in Theorem 1 .

In $\S 2$ we shall show that Theorem 2 implies Theorem 1. The proof of Theorem 2 consists in showing that the hypothesis (1.8) implies that $q_{1}-q_{2} \in C^{0}(\Omega)$. This is done by using an extension of the special solutions constructed in [S-U, II] to potentials with jump type singularities across a submanifold.

Isakov [I] has considered earlier the inverse transmission problem and obtained several interesting uniqueness results. However, his methods do not apply here.

The plan of this paper is as follows: In $\S 2$ we shall prove Theorem 1 and Theorem 2, and in $\S 3$ we shall prove a technical proposition needed in $\S 2$.

\section{Proofs of THE THEOREMS}

First we show how to prove Theorem 1 using Theorem 2. It is well known by now how to relate the Dirichlet to Neumann map $\Lambda_{q-\lambda_{0}^{2}}$ with the fixed energy scattering amplitude $a\left(\lambda_{0}, \theta, \omega\right)$ in any dimension. We sketch the procedure below. We assume $q \in L^{\infty}\left(\mathbb{R}^{n}\right)$, supp $q \subseteq B(0, R)$. Let $G_{q}\left(x, y, \lambda_{0}\right)$ be the outgoing Green's kernel for $-\Delta+q-\lambda_{0}^{2}$. The single-layer operator, which is an invertible operator from $H^{1 / 2}(\partial \Omega)$ to $H^{3 / 2}(\partial \Omega)$, is defined by

$$
\mathscr{S}_{q-\lambda_{0}^{2}} f(x)=\int_{\partial B(0, R)} G_{q}\left(x, y, \lambda_{0}\right) f(y) d S
$$

where $d S$ denotes surface measure.

It was proven in [N] (see Theorem 1.6; the proof is also valid in two dimensions) that

$$
\Lambda_{q-\lambda_{0}^{2}} \rightarrow \mathscr{S}_{q-\lambda_{0}^{2}}
$$

is injective. More precisely (see (1.40) in $[\mathrm{N}])$

$$
\Lambda_{q-\lambda_{0}^{2}}=\Lambda_{-\lambda_{0}^{2}}+\mathscr{S}_{q-\lambda_{0}^{2}}^{-1}-\left(\mathscr{S}_{\lambda_{0}}^{+}\right)^{-1}
$$

where $\mathscr{S}_{q-\lambda_{0}^{2}}^{+}$is as in (2.1) with $q=0$. Next we sketch how to prove that the map

$$
\mathscr{S}_{q-\lambda_{0}^{2}} \rightarrow \mathscr{A}_{\lambda_{0}}(q)
$$

is injective. 
This is an old result of Berezanskii [B] who showed how to go from the far field $\left(\mathscr{A}_{\lambda_{0}}(q)\right)$ to the near field $\left(\mathscr{S}_{q-\lambda_{0}^{2}}\right)$. The main element is the asymptotic expansion of the outgoing Green's kernel, namely

$$
G_{q}\left(x, y, \lambda_{0}\right)=\frac{e^{i \lambda_{0}|x|}}{|x|^{(n-1) / 2}} \psi_{+}\left(\lambda_{0}, y, \theta\right)+O\left(|x|^{-(n-1) / 2-1}\right)
$$

with $\theta=-x /|x|$ and $\psi_{+}$the outgoing eigenfunction. Now if $\mathscr{A}_{\lambda_{0}}\left(q_{1}\right)=$ $\mathscr{A}_{\lambda_{0}}\left(q_{2}\right)$, by $(2.5)$ and $(1.1)$ we get

$$
G_{q_{1}}\left(x, y, \lambda_{0}\right)-G_{q_{2}}\left(x, y, \lambda_{0}\right)=O\left(|x|^{-(n-1) / 2-1}|y|^{-(n-1) / 2-1}\right) .
$$

Now

solves

$$
\varphi(x, y)=G_{q_{1}}\left(x, y, \lambda_{0}\right)-G_{q_{2}}\left(x, y, \lambda_{0}\right)
$$

$$
\left(-\Delta_{x}-\lambda_{0}^{2}\right) \varphi=0 \text { for }|x| \geq R,|y| \geq R .
$$

Therefore by Rellich's lemma we obtain that

$$
G_{q_{1}}\left(x, y, \lambda_{0}\right)=G_{q_{2}}\left(x, y, \lambda_{0}\right) \text { for }|x|,|y| \geq R
$$

proving the injectivity of the map (2.4). 2.

In this way, we have reduced the proof of Theorem 1 to the proof of Theorem

Theorem 2 follows readily from the following

Proposition 2.1. Assume $\Omega, \lambda_{0}, \Omega_{0}^{j}, q_{j}, j=1,2$, as in Theorem 2 with $\Lambda_{q_{1}-\lambda_{0}^{2}}=\Lambda_{q_{2}-\lambda_{0}^{2}}$. Then $q_{1}-q_{2} \in C^{0}(\Omega)$.

Before giving a proof of Proposition 2.1, we need to recall the construction of special solutions of $(-\Delta+q) u=0$, where $q \in L^{\infty}$ with compact support.

Proposition 2.2 [S-U, II]. Let $\zeta \in \mathbb{C}^{2}$ with $\zeta \cdot \zeta=0, q \in L^{\infty}\left(\mathbb{R}^{2}\right)$ with compact support, and $-1<\delta<0$. Then there exists a constant $C_{1}=C_{1}(\delta)$ such that for $|\zeta|>C_{1}\left\|\left(1+|x|^{2}\right) q\right\|_{L^{\infty}\left(\mathbb{R}^{2}\right)}$, there exists a unique solution of $(-\Delta+q) u_{q}=0$ in $\mathbb{R}^{2}$ of the form

$$
u_{q}(x, \zeta)=e^{x \cdot \zeta}\left(1+\psi_{q}(x, \zeta)\right)
$$

with $\psi_{q} \in L_{\delta}^{2}\left(\mathbb{R}^{2}\right)$. Furthermore there exists a constant $C_{2}=C_{2}\left(\delta,\|q\|_{L^{\infty}\left(\mathbb{R}^{2}\right)}\right)$ such that

$$
\left\|\psi_{q}\right\|_{L_{\delta}^{2}\left(\mathbb{R}^{2}\right)} \leq C_{2} /|\zeta|
$$

Here $L_{\delta}^{2}\left(\mathbb{R}^{2}\right)$ is the weighted $L^{2}$ space:

$$
L_{\delta}^{2}\left(\mathbb{R}^{2}\right)=\left\{f ; \int_{\mathbb{R}^{2}}\left(1+|x|^{2}\right)^{\delta}|f(x)|^{2} d x<\infty\right\} .
$$

If we choose

$$
\zeta=\frac{1}{2}(i k+J k), \quad k=\left(k_{1}, k_{2}\right) \in \mathbb{R}^{2}, J=\left(\begin{array}{cc}
0 & 1 \\
-1 & 0
\end{array}\right), i=\sqrt{-1},
$$

then a straightforward computation shows that

$$
\bar{\partial}\left(\partial+\left(k_{2}+i k_{1}\right)\right) \psi_{q}-q \psi_{q}=q .
$$

The above proposition follows directly from the lemma below. 
Lemma 2.1 [S-U, II]. Let $g \in L^{\infty}\left(\mathbb{R}^{2}\right)$, with compact support and $f \in L_{\delta+1}^{2}\left(\mathbb{R}^{2}\right)$, $-1<\delta<0$, and $l \in \mathbb{C}$. Then there exists a constant $C_{3}=C_{3}(\delta)$ such that for $|l|>C_{3}\left\|\left(1+|x|^{2}\right) g\right\|_{L^{\infty}\left(\mathbb{R}^{2}\right)}$ there exists a unique function $\omega \in L_{\delta}^{2}\left(\mathbb{R}^{2}\right)$ satisfying

$$
\bar{\partial}(\partial+l) \omega-g \omega=f \quad \text { in } \mathbb{R}^{2} .
$$

Moreover

$$
\|\omega\|_{L_{\delta}^{2}\left(\mathbb{R}^{2}\right)} \leq\left(C_{4} /|l|\right)\|f\|_{L_{\delta+1}^{2}\left(\mathbb{R}^{2}\right)},
$$

where $C_{4}=C_{4}\left(\delta,\|g\|_{L^{\infty}\left(\mathbb{R}^{2}\right)}\right)$ is a constant.

In [S-U, II] it was also proven that if we assume in addition that $q \in C^{1}\left(\mathbb{R}^{2}\right)$ then $\psi_{q}$ has an asymptotic expansion for $|\zeta|$ large of the form

$$
\psi_{q}(x, \zeta)=\frac{a(x)}{k_{2}+i k_{1}}+\frac{b(x, k)}{\left(k_{2}+i k_{1}\right)^{2}}, \quad x \text { in } \mathbb{R}^{2},
$$

with $a, b \in L_{\delta}^{2}\left(\mathbb{R}^{2}\right),-1<\delta<0$. Moreover,

$$
\|a\|_{L_{\delta}^{2}\left(\mathbb{R}^{2}\right)}+\|b\|_{L_{\delta}^{2}\left(\mathbb{R}^{2}\right)}<C_{5}
$$

where $C_{5}=C\left(\delta,\|q\|_{C^{1}\left(\mathbb{R}^{2}\right)}\right)$ is a constant.

Here $a$ satisfies

$$
\bar{\partial} a=q
$$

and $b$ satisfies

$$
\bar{\partial}\left(\partial+k_{2}+i k_{1}\right) b-q b=k(q a-4 \partial q) .
$$

In our case the difficulty in proving an expansion of the form (2.12) is that we cannot directly use the proof in [S-U, II] to conclude $b \in L_{\delta}^{2}\left(\mathbb{R}^{2}\right)$ since the right-hand side of (2.14) is not even a function. A very similar problem was considered in [Su, II]. An expansion of the form (2.12) was proven there if $\partial q$ has delta type singularities on the boundary of simply connected smooth subdomains of $\Omega$. In our case, we need to prove such an expansion for potentials having jump type singularities across general smooth subdomain of $\Omega$, not just simply connected ones. However, this difficulty can be dealt with, as stated in the next proposition. The proof of this result will be given in the next section.

Proposition 2.3. Let $q \in L^{\infty}\left(\mathbb{R}^{2}\right)$ with compact support and $\Omega_{0} \subset \mathbb{R}^{2}$ be a bounded domain with smooth boundary. Let $q \in C^{1}\left(\bar{\Omega}_{0}\right)$, and $q \in C^{1}\left(\mathbb{R}^{2} \backslash \Omega_{0}\right)$. Let $\zeta \in \mathbb{C}^{2}$ with $\zeta \cdot \zeta=0,-1<\delta<0$. Then for $|\zeta|>C_{1}\left\|\left(1+|x|^{2}\right) q\right\|_{L^{\infty}\left(\mathbb{R}^{2}\right)}$, the unique solution of $(-\Delta+q) u=0$ as in (2.7) satisfies

$$
\psi_{q}(x, \zeta)=\frac{a(x)}{k_{2}+i k_{1}}+\frac{b(x, k)}{\left(k_{2}+i k_{1}\right)^{2}}, \quad x \text { in } \mathbb{R}^{2},
$$

with

$$
\|a\|_{L_{\delta}^{2}\left(\mathbb{R}^{2}\right)}+\|b(x, k)\|_{L_{\delta}^{2}\left(\mathbb{R}^{2}\right)}<C_{6}
$$

and $C_{6}=C_{6}\left(\delta,\|q\|_{C^{1}\left(\bar{\Omega}_{0}\right)},\|q\|_{C^{1}\left(\mathbb{R}^{2} \backslash \Omega_{0}\right)}\right)$ is a constant.

Given $q \in L^{\infty}(\Omega)$ and the corresponding special solution $\psi_{q}$ as in (2.4) (we extend $q=0$ outside $\Omega$ ). We consider the function $T_{q}$ considered in the 
$\bar{\partial}$ approach to the inverse scattering problem by Beals and Coifman [B-C] and Ablowitz and Nachman [N-A], which is defined by

$$
T_{q}(k)=\int_{\Omega} e^{i x \cdot k} q(x)\left(1+\psi_{q}\left(x, \xi_{k}\right)\right) d x
$$

where $k=\left(k_{1}, k_{2}\right) \in \mathbb{R}^{2},|k|$ large and

$$
\xi_{k}=\frac{1}{2}(J k+i k), \quad J=\left(\begin{array}{cc}
0 & 1 \\
-1 & 0
\end{array}\right) .
$$

An important fact about $T_{q}$ is that knowledge of $\Lambda_{q}$ determines $T_{q}$ uniquely as a function of $k$ for $|k|$ large (see [N-S-U]). The proof of this fact follows readily by integrating by parts and a result in [S-U, II]. Namely, $\psi_{q}\left(x, \xi_{k}\right)$ satisfies

$$
\Delta \psi_{q}+2 \xi_{k} \cdot \nabla \psi_{q}=q \psi_{q}+q .
$$

Multiplying (2.18) by $e^{i x \cdot k}$, integrating both sides of (2.15) over $\Omega$, and using Green's theorem we get

$$
\begin{aligned}
T_{q}(k) & =\int_{\Omega} e^{i x \cdot k} q(x)\left(1+\psi_{q}\left(x, \xi_{k}\right)\right) d x \\
& =\int_{\partial \Omega}\left[e^{i x \cdot k} \frac{\partial \psi_{q}}{\partial \nu}-\psi_{q} \frac{\partial}{\partial \nu}\left(e^{i x \cdot k}\right)+2 e^{i x \cdot k}\left(\xi_{k} \cdot \nu\right) \psi_{q}\right] d S .
\end{aligned}
$$

Thus, $T_{q}(k)$ depends only on the boundary values of $\psi_{q}$ and of $\partial \psi_{q} / \partial \nu$. Applying Proposition 2.1 of [S-U, II] we have that these boundary values are determined uniquely by $\Lambda_{q}$. Therefore $T_{q}$ is determined uniquely by $\Lambda_{q}$.

In the proof below we shall show that $T_{q}$ contains all the information about the location of the singularity of $q$ as well as the jump of $q$ across the curve of discontinuity. A very interesting problem is to reconstruct the location of singularities of the potential and the jump across the surface of discontinuity from $T_{q}$.

Proof of Theorem 2 using Proposition 2.3. Let $R=C_{1}\left\|\left(1+|x|^{2}\right) q\right\|_{L^{\infty}}$ where $C_{1}$ is as in Proposition 2.2. We assume $R>1$. We shall show that

$$
|k|^{s}\left(T_{q}(k)-\hat{q}(k)\right) \in L^{2}(|k|>R), \quad 0 \leq s<1 .
$$

By (2.19),

$$
T_{q}(k)-\hat{q}(k)=\int_{\Omega} e^{i x \cdot k} q \psi_{q}\left(x, \xi_{k}\right) d x .
$$

By Proposition 2.3 we have that

$$
\begin{aligned}
|k|^{s}\left|\int_{\Omega} e^{i x \cdot k} q \psi_{q}\left(x, \xi_{k}\right) d x\right| \leq & \frac{1}{|k|^{1-s}}\left|\int_{\Omega} e^{i x \cdot k} q(x) a(x) d x\right| \\
& +\frac{1}{|k|^{2-s}} \int_{\Omega}|q(x) b(x, k)| d x .
\end{aligned}
$$


Thus we get

$$
\begin{aligned}
& \left(\int_{|k|>R}|k|^{2 s}\left|T_{q}(k)-\hat{q}(k)\right|^{2} d k\right)^{1 / 2} \leq\left\|\int_{\Omega} e^{i x \cdot k} q(x) a(x) d x\right\|_{L^{2}\left(\mathbb{R}^{2}\right)} \\
& \quad+\left(\int_{|k|>R}\left(\frac{1}{|k|^{2-s}}\right)^{2}\left(\int_{\Omega}|q(x) b(x, k)| d x\right)^{2} d k\right)^{1 / 2} \\
& =I_{1}+I_{2} .
\end{aligned}
$$

By using Plancherel's Theorem we obtain

$$
I_{1}=\left\|\int_{\mathbb{R}^{2}} e^{i x \cdot k} q(x) a(x) d x\right\|_{L^{2}\left(\mathbb{R}^{2}\right)}=(2 \pi)^{2}\|q(x) a(x)\|_{L^{2}\left(\mathbb{R}^{2}\right)}
$$

and by the estimate (2.12) on $a(x)$ we get

$$
I_{1} \leq\|q\|_{L^{\infty}(\Omega)}\|a(x)\|_{L_{\delta}^{2}\left(\mathbb{R}^{2}\right)}<+\infty .
$$

Using the estimate on $b(x, k)$ in Proposition 2.3 we obtain

$$
I_{2} \leq\|q\|_{L^{\infty}(\Omega)}\left(\int_{|k|>R}\left(\frac{1}{|k|^{2-s}}\right)^{2}\|b(\cdot, k)\|_{L^{2}(\Omega)}^{2} d k\right)^{1 / 2} \leq C\|q\|_{L^{\infty}(\Omega)}<\infty
$$

for some constant $C<\infty$. Thus, we have proven (2.20). Now let $q_{j}, j=1,2$, be given satisfying the hypotheses of Theorem 2 . The assumption $\Lambda_{q_{1}}=\Lambda_{q_{2}}$ implies

$$
T_{q_{1}}(k)=T_{q_{2}}(k) \text { for }|k| \text { large. }
$$

Thus

and by $(2.20)$

$$
\hat{q}_{1}(k)-\hat{q}_{2}(k)=\int_{\Omega} e^{i x \cdot k} q_{2} \psi_{q_{2}} d x-\int_{\Omega} e^{i x \cdot k} q_{1} \psi_{q_{1}} d x
$$

$$
|k|^{s}\left(\hat{q}_{1}(k)-\hat{q}_{2}(k)\right) \in L^{2}(|k|>R)
$$

for $0 \leq s<1$. This implies that

$$
\hat{q}_{1}(k)-\hat{q}_{2}(k) \in L_{s}^{2}\left(\mathbb{R}^{2}\right)
$$

and, consequently,

$$
q_{1}(x)-q_{2}(x) \in H^{s}(\Omega) \text { for all } s, 0 \leq s<1 .
$$

To prove that $q_{1}$ and $q_{2}$ have the same location of singularity as well as jump across the discontinuities, it is enough to show that

$$
q_{1}-q_{2} \in C^{0}(\Omega) \text {. }
$$

This follows from (2.18) and the following trace lemma.

Lemma 2.2. Let $h$ be a piecewise $C^{1}$ function defined on $\Omega$. Suppose $h \in$ $H^{s}(\Omega), s>1 / 2$. Then $h \in C^{0}(\Omega)$.

Proof. Suppose $h$ is not in $C^{0}(\Omega)$. Since $h$ is piecewise $C^{1}$ there exists a disc $B \subset \Omega$ and a simple $C^{1}$ curve $L \subset B$ such that when we restrict $h$ on $B, h$ is $C^{1}$ on $B \backslash L$ and $h$ has jump type discontinuities on $L$. We may assume, without loss of generality that $B=\{|x| \leq \varepsilon\}$ and $L$ is a portion of the $x_{1}$-axis in $B$. Furthermore, by multiplying $h$ by a suitable compactly 
supported, positive function, one may assume further that $h$ itself is supported in $B$. Now consider the function:

$$
F: x_{2} \rightarrow h\left(\cdot, x_{2}\right) \in L^{2}\left(\mathbb{R}_{x_{1}}\right), \quad x_{2} \neq 0,
$$

where $h\left(\cdot, x_{2}\right)$ is defined as a function of $x_{1}$. By assumption, $h \in H^{s}\left(\mathbb{R}^{2}\right)$, $s>1 / 2$. Thus by the standard trace theorem, $F$ must be continuous as a map from $\mathbb{R}_{x_{2}}$, to $L^{2}\left(\mathbb{R}_{x_{1}}\right)$. But this is not the case since

$$
\lim _{x_{2} \rightarrow 0^{+}} h\left(\cdot, x_{2}\right) \neq \lim _{x_{2} \rightarrow 0^{-}} h\left(\cdot, x_{2}\right) .
$$

\section{Proof of Proposition 2.3}

This section is devoted to the proof of Proposition 2.3. We shall show that the special solutions $\psi_{q}$ have expansions of the form (2.12) for $|k|$ large, where $q$ has a jump type discontinuity across the boundary of a subdomain $\Omega_{0} \subset \Omega$. The existence of such an expansion is proven in [Su, II] when $\Omega_{0}$ is a simply connected domain. In the case that $\Omega_{0}$ is not simply connected some difficulties arise and the method in [Su, II] cannot be applied directly.

First we claim that, under the hypotheses of Proposition 2.3, the function $q$ can always be written as a sum of a finite number of other functions that carry only jump type discontinuity across some simply-connected domains. We state a simple lemma describing precisely such a decomposition of $q$.

Lemma 3.1. Let $q$ be a function in $L^{\infty}(\Omega)$ such that $q \in C^{1}\left(\Omega-\Omega_{0}\right)$ and $q \in C^{1}\left(\bar{\Omega}_{0}\right)$, where $\bar{\Omega}_{0} \subset \Omega$ is a subdomain with smooth boundary. Then there exists a simply-connected domain $D_{j}$, and functions $q_{0}, q_{j}, 1 \leq j \leq m$, such that

(1) $q=q_{0}+\sum_{j=1}^{m} q_{j}$.

(2) $q_{0} \in C^{1}(\Omega)$.

(3) $\left.q_{j}\right|_{D_{j}} \in C^{1}\left(\bar{D}_{j}\right),\left.q_{j}\right|_{\Omega \backslash D_{j}} \equiv 0,1 \leq j \leq m$.

Proof. The proof of this lemma is elementary but cumbersome. We only give a detailed proof for a special case. The complete proof can be done using induction.

Let $D_{1}, D_{2}$, and $D_{3}$ be simply-connected domains with smooth boundary such that $\overline{D_{1} \cap D_{2}}=\varnothing, \bar{D}_{j} \subseteq \Omega, j=1,2$, and $\bar{D}_{3} \subset D_{2}$. We consider the case that $\Omega_{0}=D_{1} \cup\left(D_{2} \backslash D_{3}\right)$.

Let $q_{0} \in C^{1}(\Omega)$ be a $C^{1}$ extension of $\left.q\right|_{\Omega \backslash \Omega_{0}}$ to $\Omega$, i.e., $q_{0}(x)=q(x)$ for $x \in \Omega \backslash \Omega_{0}$. Then let

$$
q_{1}(x)= \begin{cases}q(x)-q_{0}(x), & x \in D_{1}, \\ 0, & x \in \Omega \backslash D_{1} .\end{cases}
$$

Next, let $q_{2}^{*} \in C^{1}\left(D_{2}\right)$ be a $C^{1}$ extension of $q(x)-\left.q_{0}(x)\right|_{D_{2} \backslash D_{3}}$ to $D_{2}$ and set

$$
q_{2}(x)= \begin{cases}q_{2}^{*}, & x \in D_{2}, \\ 0, & x \in D_{2} .\end{cases}
$$

Finally set

$$
q_{3}(x)= \begin{cases}q(x)-q_{2}(x), & x \in D_{3}, \\ 0, & x \in \Omega \backslash D_{3} .\end{cases}
$$


Clearly, $q_{0}, q_{1}, q_{2}$, and $q_{3}$ satisfy the condition of Lemma 3.1.

Hereafter, we assume $q=q_{0}+\sum_{j=1}^{m} q_{j}$ where $q_{0}, q_{j}, 1 \leq j \leq m$, are given by the above lemma. For $\psi_{q}$ to have an expansion of the form (2.2), $a$ and $b$ must satisfy

$$
\begin{gathered}
\bar{\partial} a=q, \\
\bar{\partial}\left(\partial+\left(k_{2}+i k_{1}\right)\right) b-q b=k(q a-4 \partial q) .
\end{gathered}
$$

As we mentioned earlier, the difficulty in obtaining the expansion (2.2) lies in the equation (3.2). More specifically, the discontinuity of $q$ introduces a delta type singularity in $\partial q$, while the rest of the right-hand side of (3.2) gives no problem. We divide $b$ into two parts

$$
b=b_{1}+b_{2}
$$

where

$$
\begin{gathered}
\bar{\partial}\left(\partial+\left(k_{2}+i k_{1}\right)\right) b_{1}-q b_{1}=k q a-4 k \partial q_{0}, \\
\bar{\partial}\left(\partial+\left(k_{2}+i k_{1}\right)\right) b_{2}-q b_{2}=-4 k \sum_{j=1}^{m} \partial q_{j} .
\end{gathered}
$$

By Lemma 3.1, we note that $\partial q_{0} \in L^{\infty}$ with compact support. (We define $\left(\partial q_{0}\right)(x)=0$ when $x \in \mathbb{R}^{2} \backslash \Omega$.) Then by Lemma 2.1 , there exists a solution $b_{1}$, satisfying (3.4) and

$$
\left\|b_{1}(\cdot, k)\right\|_{L_{\delta}^{2}\left(\mathbb{R}^{2}\right)} \leq C
$$

where $C$ is independent of $k$.

To solve (3.5), we divide $b_{2}$ into two parts again:

$$
b_{2}=b_{3}+b_{4}
$$

where

$$
\begin{gathered}
\bar{\partial}\left(\partial+\left(k_{2}+i k_{1}\right)\right) b_{3}=4 k \sum_{j=1}^{m} \partial q_{j}, \\
\bar{\partial}\left(\partial+\left(k_{2}+i k_{1}\right)\right) b_{4}-q b_{4}=q b_{3} .
\end{gathered}
$$

The crux of the matter is to solve (3.8). Suppose for the moment that one gets a solution $b_{3}$ in $L_{\delta}^{2}\left(\mathbb{R}^{2}\right)$ for (3.8). Then by Lemma 2.4 again, there will be a solution $b_{4}$ for (3.9) and the whole problem is solved.

Let $l$ be a simple closed and smooth curve in $\mathbb{R}^{2}$, and let $h \in L^{\infty}(l)$. We define the distribution $\delta_{h, l} \in D^{\prime}\left(\mathbb{R}^{2}\right)$ by

$$
\delta_{h, l}(\varphi)=\int_{l} h(\tau) \varphi(\tau) d \tau, \quad \forall \varphi \in C_{0}^{\infty}\left(\mathbb{R}^{2}\right) .
$$

Let $f$ be a function on $\mathbb{R}^{2}$ such that $f$ is $C^{1}$ everywhere except in $l$, where $f$ has a jump type discontinuity across $l$. We denote by $[f]_{l}$ its jump, that is

$$
[f]_{l}(\sigma)=\lim _{\substack{x \rightarrow \sigma \\ \text { from inside } l}} f(x)-\lim _{\substack{x \rightarrow \sigma \\ \text { from outside } l}} f(x) .
$$


Using these notations we can write

$$
\partial q_{j}=\delta_{\left(\nu\left(x_{1}\right)-i \nu\left(x_{2}\right)\right)\left[q_{j}\right] / 2, \partial D_{j}}+p_{j}, \quad 1 \leq j \leq m,
$$

where $\nu\left(x_{1}\right), \nu\left(x_{2}\right)$ denote the $x_{1}$ and $x_{2}$ components of $\nu$ and

$$
p_{j}= \begin{cases}\partial q_{j}, & x \in D_{j} \text { or } \mathbb{R}^{2} \backslash \bar{D}_{j}, \\ 0, & x \in \partial D_{j},\end{cases}
$$

and $z=x_{1}+i x_{2}$. Then we split $b_{3}$ into

$$
b_{3}=\tilde{b}_{3}+\sum_{j=1}^{m} b_{3}^{j}
$$

where

$$
\begin{gathered}
\bar{\partial}\left(\partial+\left(k_{2}+i k_{1}\right)\right) \tilde{b}_{3}=4 k \sum_{j=1}^{m} p_{j}, \\
\bar{\partial}\left(\partial+\left(k_{2}+i k_{1}\right)\right) b_{3}^{j}=4 k \delta_{\left(\nu\left(x_{1}\right)-i \nu\left(x_{2}\right)\right)\left[q_{j}\right] / 2, \partial D_{j}}, \quad 1 \leq j \leq m .
\end{gathered}
$$

From (3.12) we conclude $p_{j} \in L^{\infty}(\Omega)$, thus we can get a solution for (3.14) using Lemma 2.4. The existence of a solution for (3.15) follows directly from the proposition below.

Proposition 3.1. Let $l$ be a simple closed and smooth curve in $\mathbb{R}^{2}$ and let $h \in$ $H^{1}(l)$. There exists a unique function $\omega$ in $L_{\delta}^{2}\left(\mathbb{R}^{2}\right),-1<\delta<0$, satisfying

$$
\bar{\partial}\left(\partial+\left(k_{1}+i k_{2}\right)\right) \omega=\delta_{h, l}
$$

provided that $k=k_{1}+i k_{2} \neq 0$. Moreover

$$
\|\omega\|_{L_{\delta}^{2}\left(R^{2}\right)} \leq(C /|k|)\|h\|_{L^{2}(l)}^{1 / 2}\|h\|_{H^{1}(l)}^{1 / 2} .
$$

Proof. The proof of this proposition is similar to the proof of Lemma 5.1 in [Su, II]. We only give an outline of the proof. Let us recall two lemmas. Let $L$ be either $\partial$ or $\bar{\partial}$.

Lemma 3.2 [S-U, II]. Let $f \in L_{\delta+1}^{2}\left(\mathbb{R}^{2}\right),-1<\delta<0$. Then there exists a unique solution $u \in L_{\delta}^{2}\left(\mathbb{R}^{2}\right)$ satisfying

$$
L u=f .
$$

Moreover

$$
\|u\|_{L_{\delta}^{2}\left(\mathbb{R}^{2}\right)}+\|\nabla u\|_{L_{\delta+1}^{2}\left(\mathbb{R}^{2}\right)} \leq C\|f\|_{L_{\delta+1}^{2}\left(\mathbb{R}^{2}\right)},
$$

where $C=C(\delta)$ is a constant.

Lemma 3.3 [Su, II]. Let $l=\left\{x \in \mathbb{R}^{2},|x|=1\right\}, h \in H^{1}(l),-1<\delta<0$. Then there exists a unique $v \in L_{\delta}^{2}\left(\mathbb{R}^{2}\right)$ satisfies

$$
L v=\delta_{f, l} \text {. }
$$

Moreover,

$$
\|v\|_{L_{\delta}^{2}\left(R^{2}\right)} \leq C\|f\|_{L^{2}(l)},
$$




$$
\|\nabla v\|_{L^{2}(|x|<1)}+\|\nabla v\|_{L_{\delta^{\prime} 1}^{2}(|x|>1)} \leq C\|f\|_{L^{2}(l)}^{1 / 2}\|f\|_{H^{1}(l)}^{1 / 2} .
$$

We construct

$$
\omega=\frac{1}{k}\left(A+e^{-2 i \operatorname{Im}(k z)} B\right) .
$$

A computation shows that $\omega$ solves (3.16) if

$$
\begin{gathered}
\bar{\partial} A=\delta_{h, l}, \\
\partial B=-e^{2 i \operatorname{Im}(k z)} \partial A .
\end{gathered}
$$

By a conformal change of variables, we may assume that $l$ is the unit circle of $\mathbb{R}^{2}$. (Note that $\bar{\partial}$ and $\partial$ is invariant under such a change of variables.) Using Lemma 3.3 we get a unique solution $A$ satisfying

$$
\|A\|_{L_{\delta}^{2}\left(R^{2}\right)}+\|\nabla A\|_{L_{\delta+1}^{2}\left(R^{2} \backslash l\right)} \leq C\|h\|_{L^{2}(l)}^{1 / 2}\|h\|_{H^{1}(l)}^{1 / 2} .
$$

To prove (3.20), we note that (assuming $l$ is the unit circle) $\partial A=\delta_{(\bar{z} / z) h, l}+A_{1}$, where

$$
A_{1}= \begin{cases}\partial A, & z \in \mathbb{R}^{2} \backslash l \\ 0, & z \in l\end{cases}
$$

is a function in $L_{\delta+1}^{2}\left(\mathbb{R}^{2}\right)$. We write

$$
B=B_{1}+B_{2},
$$

where

$$
\begin{gathered}
\partial B_{1}=-e^{2 i \operatorname{Im}(k z)} \delta_{(\bar{z} / z) h, l}=\delta_{\left[-e^{2 i \operatorname{Im}(k z)(\bar{z} / z) h], l},\right.}, \\
\partial B_{2}=A_{1} .
\end{gathered}
$$

By using Lemma 3.3, we get a solution $B_{1} \in L_{\delta}^{2}\left(\mathbb{R}^{2}\right)$ and

$$
\left\|B_{1}\right\|_{L_{\delta}^{2}\left(R^{2}\right)} \leq C\|h\|_{L^{2}(l)}^{1 / 2}\|h\|_{H^{1}(l)}^{1 / 2} .
$$

Since $A_{1} \in L_{\delta+1}^{2}\left(\mathbb{R}^{2}\right)$ is a regular function, one can solve (3.28) by using Lemma 3.3 to get a solution $B_{2}$ satisfying

$$
\left\|B_{2}\right\|_{L_{\delta}^{2}\left(\mathbb{R}^{2}\right)} \leq C\|\nabla A\|_{L_{\delta+1}^{2}\left(\mathbb{R}^{2} \backslash l\right)} .
$$

Combining the estimates (3.26), (3.30), and (3.31) one gets the estimate (3.17).

The existence of the expansion (3.2) is now proven. Uniqueness follows from Lemma 2.1.

\section{ACKNOWLEDGMENT}

The authors would like to thank V. Isakov and J. Sylvester for helpful discussions of the results, and A. Nachman for helpful discussions on the relation between the far field and the near field and for his reference to [B].

Note added in proof. We have recently generalized Theorems 1 and 2 to a more general class of singular potentials, see [Su-U, III]. 


\section{REFERENCES}

[B] Y. Berezanskii, The uniqueness theorem in the inverse problem of spectral analysis for the Schrödinger operator, Trudy Moskov. Mat. Obshch. 7 (1958), 1-62.

[B-C] R. Beals and R. Coifman, Multidimensional inverse scattering and nonlinear PDE, Proc. Sympos. Pure Math., vol. 43, Amer. Math. Soc., Providence, R.I., 1985, pp. 45-70.

[I] V. Isakov, On uniqueness in the inverse transmission scattering problem, Comm. Partial Differential Equations 15 (1990), 1565-1587.

[N] A. Nachman, Reconstructions from boundary measurements, Ann. of Math. (2) 128 (1988), 531-587.

[N-A] A. Nachman and M. Ablowitz, A multidimensional inverse scattering method, Stud. Appl. Math. 71 (1984), 243-250.

[N-S-U] A. Nachman, J. Sylvester, and G. Uhlmann, An n-dimensional Borg-Levinson theorem, Comm. Math. Phys. 115 (1988), 595-605.

[No] R. Novikov, Multidimensional inverse spectral problems for the equation $-\Delta \psi+(v(x)-$ $E u(x)) \psi=0$, Funktsional. Anal. i Prilozhen. 22 (1988), no. 4, 11-12; English transl. in Functional Anal. Appl. 22 (1988), no. 4, 263-272.

[Su, I] Z. Sun, On an inverse boundary value problem in two dimensions, Comm. Partial Differential Equations 14 (1989), 1101-1113.

[Su, II] _ The inverse conductivity problem in two dimensions, J. Differential Equations 87 (1990), 227-255.

[Su-U, I] Z. Sun and G. Uhlmann, Generic uniqueness for determined inverse problems in 2 dimensions, Satellite Conf. Proc., ICM 90, Springer-Verlag, 1991, pp. 145-152.

[Su-U, II] __, Generic uniqueness for an inverse boundary value problem, Duke Math. J. 82 (1991), 131-155.

[Su-U, III] Z. Sun and G. Uhlmann, Recovery of singularities for formally determined inverse problems, Comm. Math. Phys. (to appear).

[S-U, I] J. Sylvester and G. Uhlmann, A global uniqueness theorem for an inverse boundary value problem, Ann. of Math. (2) 125 (1987), 153-169.

[S-U, II] __, A uniqueness theorem for an inverse boundary value problem in electrical prospection. Comm. Pure. Appl. Math. 39 (1986), 91-112.

Department of Mathematics and Statistics, Wichita State University, Wichita, KANSAS 67208

E-mail address: sun@twsuvm.bitnet

Department of Mathematics, University of Washington, Seattle, Washington 98195 E-mail address: gunther@math.washington.edu 